\title{
Approaches for Genetic Discoveries in the Skin Commensal and Pathogenic Malassezia Yeasts
}

\author{
Giuseppe laniri ${ }^{1 *}$ and Joseph Heitman ${ }^{2}$ \\ ${ }^{1}$ Department of Agricultural, Environmental and Food Sciences, Università degli Studi del Molise, Campobasso, Italy, \\ ${ }^{2}$ Department of Molecular Genetics and Microbiology, Duke University Medical Center, Durham, NC, United States
}

\section{OPEN ACCESS}

Edited by:

Anuradha Chowdhary,

University of Delhi, India

Reviewed by:

Won Hee Jung,

Chung-Ang University, South Korea Vishukumar Aimanianda,

Institut Pasteur, France

${ }^{*}$ Correspondence:

Giuseppe lanir

giuseppe.ianiri@unimol.it

Specialty section:

This article was submitted to

Fungal Pathogenesis,

a section of the journal

Frontiers in Cellular and Infection

Microbiology

Received: 06 April 2020

Accepted: 25 June 2020

Published: 07 August 2020

Citation:

laniri G and Heitman J (2020) Approaches for Genetic Discoveries in the Skin Commensal and Pathogenic Malassezia Yeasts.

Front. Cell. Infect. Microbiol. 10:393. doi: 10.3389/fcimb.2020.00393
Malassezia includes yeasts belong to the subphylum Ustilaginomycotina within the Basidiomycota. Malassezia yeasts are commonly found as commensals on human and animal skin. Nevertheless, Malassezia species are also associated with several skin disorders, such as dandruff/seborrheic dermatitis, atopic eczema, pityriasis versicolor, and folliculitis. More recently, associations of Malassezia with Crohn's disease, pancreatic ductal adenocarcinoma, and cystic fibrosis pulmonary exacerbation have been reported. The increasing availability of genomic and molecular tools have played a crucial role in understanding the genetic basis of Malassezia commensalism and pathogenicity. In the present review we report genomics advances in Malassezia highlighting unique features that potentially impacted Malassezia biology and host adaptation. Furthermore, we describe the recently developed protocols for Agrobacterium tumefaciens-mediated transformation in Malassezia, and their applications for random insertional mutagenesis or targeted gene replacement strategies.

Keywords: Malassezia, genomics, Agrobacterium tumefaciens-mediated transformation (AMT), insertional mutagenesis, targeted gene replacement

\section{MALASSEZIA YEASTS AS COMMENSALS AND PATHOGENS}

Malassezia includes a monophyletic genus of yeasts that are the main fungal species resident on human skin and hair, representing more than $90 \%$ of the eukaryotic components of the skin microbiome (Findley et al., 2013). To date, 18 species of Malassezia have been identified (Theelen et al., 2018). The limited number of species isolated so far most likely reflects the difficulties in cultivating Malassezia under laboratory conditions, given their ability to grow in vitro only in the presence of exogenous lipids, and at a narrow range of temperatures. As commensal organisms living on the skin, Malassezia globosa, Malassezia restricta, and Malassezia sympodialis are the most common species found in humans, followed by Malassezia furfur, Malassezia yamatoensis, Malassezia dermatis, Malassezia obtusa, Malassezia japonica, and Malassezia arunalokei. Malassezia pachydermatis is mainly found in dogs and cats, Malassezia slooffiae in pigs and cats, Malassezia nana in cats and horses, Malassezia caprae in goats, Malassezia equina in horses, Malassezia cuniculi in rabbits, Malassezia brasiliensis and Malassezia psittaci in parrots, and Malassezia vespertiliones in hibernating bats (Theelen et al., 2018; Guillot and Bond, 2020). Aside from their commensal lifestyle, Malassezia yeasts are associated with a number of skin disorders, the most common of which are dandruff/seborrheic dermatitis, atopic eczema, pityriasis versicolor, and folliculitis. Occasionally, in immunocompromized hosts or patients receiving total parenteral nutrition, M. furfur, $M$. sympodialis, and M. pachydermatis can also cause systemic disease (Gaitanis et al., 2012; Saunders et al., 2012; Velegraki et al., 2015; Theelen et al., 2018; Guillot and Bond, 2020). Moreover, novel 
studies have linked Malassezia yeasts with Crohn's disease in patients with an $\mathrm{S} 12 \mathrm{~N}$ polymorphism in the gene encoding CARD9, a signaling adaptor critical for innate antifungal immunity (Limon et al., 2019), with pathogenesis of pancreatic ductal adenocarcinoma through activation of the MBL pathway (Aykut et al., 2019), and with cystic fibrosis pulmonary exacerbation (Soret et al., 2020).

\section{EVOLUTIONARY TRAJECTORY OF MALASSEZIA GENOMES CORRELATES WITH PATHOGENICITY AND NICHE ADAPTATION}

In the last decade several groups contributed to generate genomics data for the majority of species within the Malassezia genus. A GenBank search (last accessed on March 7th, 2020) finds 45 genome assemblies that include 15 known Malassezia species (Xu et al., 2007; Gioti et al., 2013; Triana et al., 2015; Wu et al., 2015; Park et al., 2017; Zhu et al., 2017; Lorch et al., 2018; Cho et al., 2019; Morand et al., 2019; Sankaranarayanan et al., 2020). Analysis of the genomes available contributed to resolve Malassezia taxonomy, and shed light on the evolutionary trajectory of pathogenesis and niche adaptation of this unusual fungal genus.

Taxonomically Malassezia are included in the subdivision Ustilaginomycotina within the Basidiomycota phylum, which also includes human and plant pathogens (Wang et al., 2014; $\mathrm{Wu}$ et al., 2015). Surprisingly, from a phylogenetic viewpoint Malassezia fungi are more closely related to the basidiomycete plant pathogen Ustilago maydis than the human pathogen Cryptococcus neoformans, and are very divergent from other fungi that are found on the skin, such as the dermatophytes and Candida albicans (Xu et al., 2007; Saunders et al., 2012; Wu et al., 2015). Within the Malassezia genus we found three clades that include two sister clades, clade $\mathrm{A}$ and clade $\mathrm{B}$, with clade $\mathrm{A}$ including subclades $\mathrm{A} 1$ and $\mathrm{A} 2$, and clade $\mathrm{C}$ that includes earlydivergent species (Figure 1A). Phylogenetic relationships of the tree of Figure 1A based on D1D2 domains of LSU rDNA agree with the previous phylogenomics data (Wu et al., 2015; de Hoog et al., 2017; Lorch et al., 2018; Theelen et al., 2018).

All haploid Malassezia species have small and compact genomes compared to other phylogenetically related fungi (7-9 Mb compared to $\sim 20 \mathrm{Mb}$ ) (Figure 1B), with genes being arranged very close to each other, and containing very short introns. At the karyotype level, haploid Malassezia species have from 6 to 9 chromosomes, based on pulsed-field gel electrophoresis (PFGE) and telomere-to-telomere genome assemblies generated with PacBio long-read sequencing technology (Boekhout and Bosboom, 1994; Boekhout et al., 1998; Sankaranarayanan et al., 2020). Using a combination of genomics, biochemical, cell biology, and molecular genetics techniques (described later in the text), Sankaranarayanan and colleagues elucidated the mechanisms of karyotype evolution within the Malassezia genus. In particular, the authors proposed an ancestral state of 9 chromosomes and two distinct mechanisms of chromosome number reduction that involve newly-identified AT-rich, fragile, centromeres: a chromosome breakage followed by loss of centromere that gave rise to 8 chromosomes in $M$. sympodialis and closely related species; and centromere inactivation accompanied by changes in DNA sequence following chromosome-chromosome fusion that gave rise to 7 chromosomes in $M$. furfur (Sankaranarayanan et al., 2020). It is intriguing to note that species with 9 chromosomes, such as $M$. globosa and $M$. restricta, are difficult to isolate and replicate in axenic conditions, while $M$. sympodialis and $M$. furfur are more readily cultivated.

At the gene level, comparative genomics revealed extensive turnover events, with significant gene loss and gene gain. Some Malassezia species have lost nearly 800 genes and have $<4,000$ predicted genes. All species have lost genes for lipid metabolism, including fatty acid synthase, $\Delta 9$-desaturase, and $\Delta^{2,3}$-enoylCoA isomerase, hence explaining Malassezia lipid dependency (Figure 2); M. pachydermatis has also lost the genes for lipid metabolism but is the only known Malassezia species that is able to grow in vitro without the addition of exogenous lipids (Figure 2); however, a recent study identified some $M$. pachydermatis isolates that are unable to grow in synthetic medium without lipids (Puig et al., 2017). Other major groups of lost genes include those encoding glycosyl hydrolases and enzymes involved in carbohydrate metabolism, concordant with the evolution of a skin-adapted fungus that uses lipids as carbon sources. Moreover, the Malassezia genomes have a low density of transposable elements, and they lack core genes of the RNA interference (RNAi) pathway, such as dicer, argonaute, and RNAdependent RNA polymerase.

Because the lack of the RNAi pathway in other fungi such as Saccharomyces cerevisiae and $U$. maydis is associated with the presence of dsRNA viruses (Drinnenberg et al., 2011), it was hypothesized that Malassezia species could also harbor mycoviruses. Corroborating this hypothesis, dsRNA mycoviruses of the Totiviridae family were found in $M$. sympodialis, $M$. globosa, M. obtusa, M. pachydermatis, M. yamatoensis, and M. restricta (Clancey et al., 2019; Park et al., 2019). In $M$. sympodialis, the viral genome includes two dsRNA elements, one of $4.6 \mathrm{~kb}$ that encodes an RNA-dependent RNA polymerase and a capsid protein, and one of $1.4 \mathrm{~kb}$ that encodes a novel unknown protein predicted to be secreted from the fungal cells and involved in host-pathogen and/or microbial interactions. Fungal cells can be cured of the mycovirus upon exposure to high temperature. Transcriptomic analysis of infected and cured strain pairs revealed that the presence of the mycovirus strongly enhances the expression of ribosomal genes, suggesting that the virus conscripts the Malassezia transcription and protein synthesis machineries. Lastly, the presence of the Malassezia mycovirus correlated with higher pathogenicity in ex vivo models (Clancey et al., 2019; Park et al., 2019).

With respect to gene gain, several unique events found in Malassezia genomes warrant consideration. First, a set of 44 Malassezia-specific gene clusters was identified, but unfortunately most of them have unknown functions that could not be predicted through bioinformatics analyses (Wu et al., 2015). One gene gain event that $\mathrm{Wu}$ and colleagues described regarded a gene with a PF06742 domain of unknown function. 


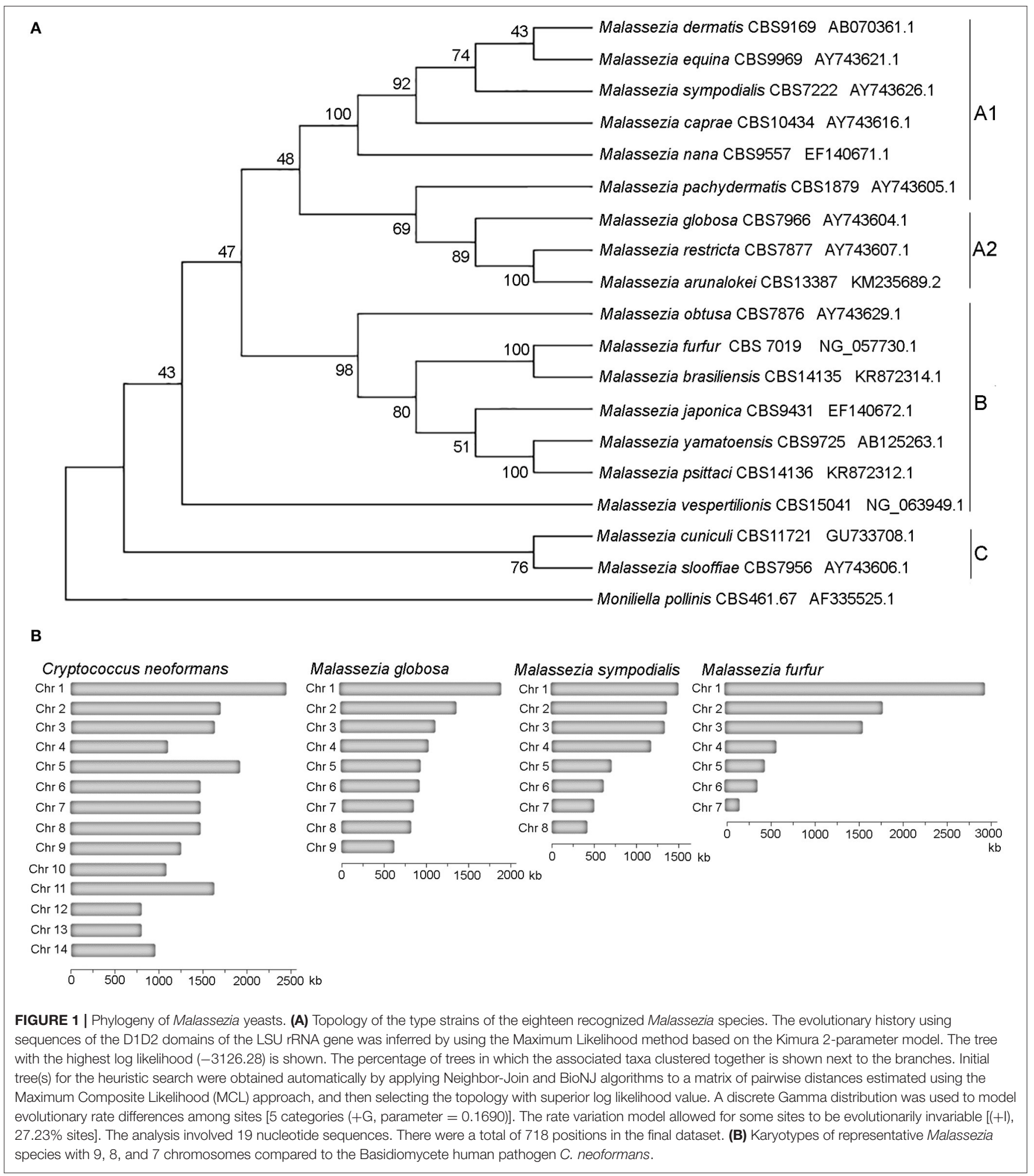

This gene is conserved in all Malassezia species and is absent in all Basidiomycota, suggesting its acquisition by a Malassezia ancestor and an important role in Malassezia evolution (Wu et al., 2015).
Second, Malassezia genomes are characterized by a significant expansion of lipase, phospholipase, peptidase, and protease gene family-encoding products predicted to break down lipids and proteins for growth, and to play roles in host and microbial 


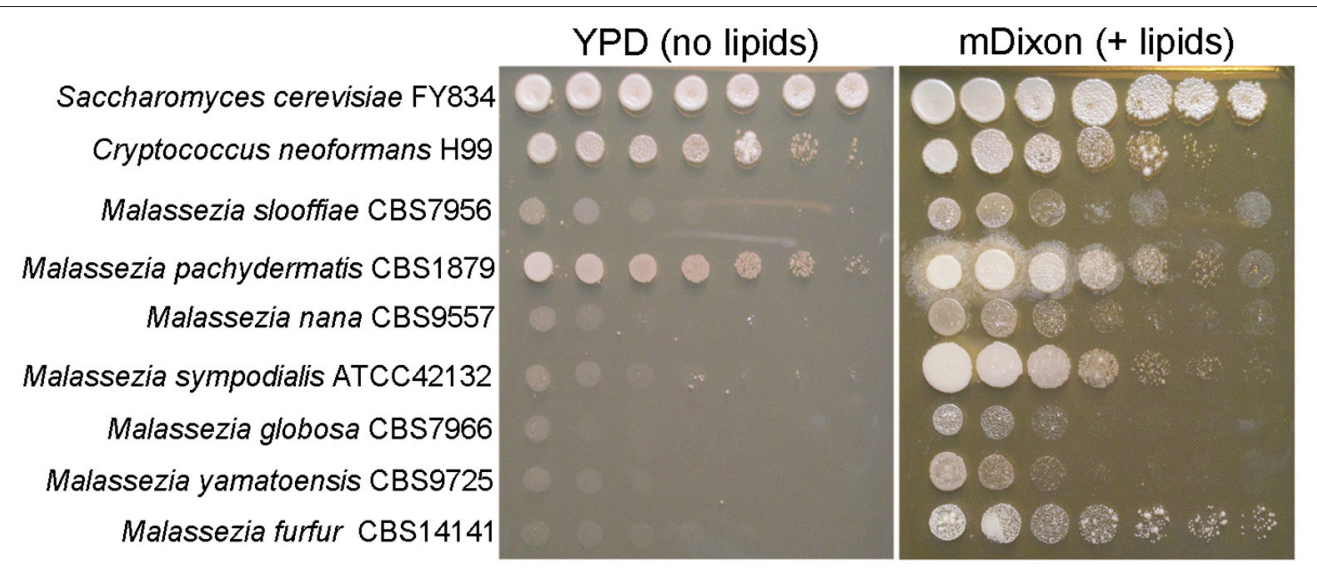

FIGURE 2 | Malassezia yeasts are lipid dependent. Ten-fold serial dilution of representative Malassezia species on medium without exogenous lipids (YPD, yeast extract $10 \mathrm{~g} / \mathrm{L}$, peptone $20 \mathrm{~g} / \mathrm{L}$, dextrose $20 \mathrm{~g} / \mathrm{L}$, agar $20 \mathrm{~g} / \mathrm{L}$ ), and on lipid-rich medium mDixon (36 g/L malt extract, $10 \mathrm{~g} / \mathrm{L}$ desiccated ox-bile, $10 \mathrm{~g} / \mathrm{L}$ mycological peptone, $2 \mathrm{ml} / \mathrm{L}$ glycerol, $10 \mathrm{ml} / \mathrm{L}$ Tween 60, agar $20 \mathrm{~g} / \mathrm{L})$.

interactions (Wu et al., 2015). Intriguingly, a similar set of enzymes is found in the genome of C. albicans, a phylogenetically distant fungus that also lives on the skin, suggesting an important role in skin colonization and niche adaptation. Moreover, analysis of the M. sympodialis and M. globosa genomes identified 89 and 169 predicted secreted proteins, most of them without any domain (Schuster et al., 2018). These predicted secreted proteins include several MalaS allergens, such as MalaS1, a $\beta$ propeller-folded protein that has fungal orthologs/homologs in some basidiomycetes and ascomycetes (Vilhelmsson et al., 2007; Gioti et al., 2013), MalaS12 that is similar to other fungal GMC oxidoreductases (Zargari et al., 2007) that play diverse roles in fungi, such as mycotoxin biosynthesis in species of Aspergillus and Penicillium (Tannous et al., 2017), and MalaS7 (in 3 copies) and MalaS8, both of which are Malassezia-specific and have unknown predicted roles (Gioti et al., 2013). Besides these, $M$. sympodialis has genes encoding six additional MalaS allergens that are conserved proteins that share high similarity with the corresponding mammalian homologs, and hence can potentially cross-react with $\mathrm{T}$ cells and induce skin inflammation (Glatz et al., 2015).

Another characteristic of Malassezia genomes is the presence of bacterial genes acquired through horizontal gene transfer (HGT) events. While the number of these events is usually limited, in Malassezia more than 30 HGT have been identified (Wu et al., 2015; Ianiri et al., 2020). HGT candidates found in the majority of the Malassezia species include genes involved in broad stress resistance, such as flavohemoglobin, catalase, and oxidoreductases, found in some cases in multiple copies. An interesting HGT candidate is the gene encoding a septicolysinlike protein, which is known as a pore-forming bacterial toxin that might play a role as virulence factor (Beceiro et al., 2013; Mosqueda et al., 2014). This gene is absent in all Malassezia species phylogenetically related to $M$. sympodialis, and is present in five copies in M. globosa. Other acquired genes encode a variety of proteins with different functions, such as hydrolysis, protein transport and folding, and detoxification of xenobiotics (Ianiri et al., 2020).

Using molecular techniques described in the section Agrobacterium tumefaciens-Mediated Transformation Enables Insertional Mutagenesis and Targeted Gene Deletion in Malassezia, we demonstrated that the HGT of the bacterial flavohemoglobin in Malassezia resulted in a gain of function critical for resistance to nitrosative stress and nitric oxide (NO) detoxification (Ianiri et al., 2020). Analysis of the available Malassezia genomes revealed additional HGT of another flavohemoglobin-encoding gene that originated from different donor bacteria. Endogenous accumulation of $\mathrm{NO}$ in the flavohemoglobin mutant results in downregulation of the allergen-encoding genes, and accordingly, we found that flavohemoglobin has a dispensable role for Malassezia pathogenesis. This study represents the first functional analysis of an HGT-acquired gene in Malassezia, and the first evaluation of a Malassezia mutant in a novel murine skin model (Sparber and LeibundGut-Landmann, 2019; Sparber et al., 2019) to assess the involvement of a Malassezia gene in pathogenesis.

\section{AGROBACTERIUM TUMEFACIENS-MEDIATED TRANSFORMATION ENABLES INSERTIONAL MUTAGENESIS AND TARGETED GENE DELETION IN MALASSEZIA}

Although the availability of sequenced genomes revealed insights about Malassezia evolution, adaptation, and gene turnover, the function of specific genes could not be studied because of the lack of transformation systems. Genetic transformation in fungi can be carried out through the combined use of lithium acetate (LiAc) and polyethylene glycol (PEG), biolistic 
bombardment, electroporation of intact cells or protoplasts, or A. tumefaciens-mediated transformation (AMT). We tested the effectiveness of these four techniques to successfully transform Malassezia, but despite several attempts, AMT was the only technique that allowed the generation of stable transformants of $M$. furfur, M. sympodialis, and M. pachydermatis (Ianiri et al., 2016; Celis et al., 2017).

A. tumefaciens is a soil-borne bacterium that has the ability to infect plants to cause a crown gall disease. The infective process is unique and relies on the natural ability of A. tumefaciens to genetically engineer host plants by introducing a short DNA fragment into their genome. The DNA fragment is called TDNA (transfer DNA) and its excision is enabled by virulence proteins induced by acetosyringone, a chemical compound that is produced by wounded plant roots and that attracts $A$. tumefaciens. The T-DNA contains genes that encode products that mimic plant hormones, and once integrated in the host genome, causes an undifferentiated growth of the plant tissues forming a tumor or gall. Researchers have exploited this natural genetic ability of $A$. tumefaciens to transfer a desired DNA molecule, usually a gene marker, into a variety of eukaryotic organisms, such as plants, animal cells, oomycetes, and fungi.

The most common use of AMT in fungal research is based on a binary vector system: one A. tumefaciens plasmid contains vir genes required for virulence (i.e., transfer of DNA into the host), and another plasmid, the $\mathrm{Ti}$ (tumor inducing) plasmid-usually it is a binary vector and is the most commonly manipulated by researchers - contains the marker gene between two 25-bp direct repeats (right and left borders, RB and LB, respectively) that define the T-DNA. The vir proteins are induced by acetosyringone and act on the T-DNA borders enabling the production of single-stranded DNA. The T-DNA is coated with proteins forming the T-complex, which is transferred into the fungal cell. The T-complex is then disassembled, and nuclear localization signals drive the translocation of the T-DNA within the fungal nucleus where integration into the genome occurs (Michielse et al., 2005) (Figure 3A). Compared to other transformation methods, AMT requires basic reagents that are common in most microbiology laboratories, and therefore it has been largely utilized for transformation of yeasts and fungi since its first use in S. cerevisiae in 1995 (Bundock et al., 1995). For more information about the method and its use in fungal biology research, there are several reviews available (Michielse et al., 2005; Frandsen, 2011; Idnurm et al., 2017).

In general, the method is straightforward: after growing the A. tumefaciens with the binary vector of interest and the fungal strain to be transformed, these two organisms are co-cultured on induction medium (IM) for a few days depending on the growth of the fungus, and subsequently transferred to a selective medium that differs based on the gene marker used (usually a dominant gene that confers resistance to an antifungal drug). A key role in the transformation process is played by the induction medium (IM), which contains acetosyringone to induce the vir genes, and it physically supports the A. tumefaciens-fungus co-culture ensuring the tight contact between the cells, which is a critical requirement for the success of the trans-kingdom conjugation process (Michielse et al., 2005).
While AMT is relatively simple in the majority of fungi, its use in Malassezia turned out to be more difficult because of the unique biology of this fungus. The first successful application of AMT was carried out in M. furfur, one of the species that displays more robust growth compared to others within the Malassezia genus (Ianiri et al., 2016). The method employed followed a previously published protocol (Ianiri et al., 2011), with the only difference being the use of a modified IM (mIM) that also included exogenous lipids (i.e., Tween and ox-bile) to favor growth of Malassezia. Stable Malassezia transformants could be generated for the first time, although the efficiency of the ATM was very low ( $<5$ transformants per transformation plate). The AMT method was then improved using a higher density of Malassezia cells, a longer co-incubation period of up to 6 days, and by performing the co-incubation step on slightly concave spots generated on nylon membranes placed on the modified IM. The latter modification was critical to facilitate cellto-cell contact between bacterial and Malassezia cells, which was otherwise hindered by the presence of Tween in the modified IM. Subsequently, the AMT protocol was further optimized by Celis et al. (2017) and Ianiri et al. (2019), as illustrated in detail in the flow charts of Figures 3B-D. Examples of representative steps of the AMT of Malassezia are shown in Figure 3E, and representative NAT-resistant $M$. furfur transformant are shown in Figure 3F. Lastly, we could never obtain transformants for $M$. globosa, a species characterized by very slow growth at a limited range of temperatures $\left(30-34^{\circ} \mathrm{C}\right)$ (unpublished data).

Several binary vectors proved to be effective for Malassezia transformation. Plasmids pAIM2 and pAIM6 were generated through fusing the ACT1 promoter and terminator of $M$. sympodialis with the NAT and NEO genes to confer resistance to nourseothricin (NAT) and neomycin sulfate G418 (NEO), respectively (Figure 4A) (Ianiri et al., 2016). Celis and colleagues successfully employed plasmid $\mathrm{pBHg}$ that includes the Escherichia coli hpt gene under the control of the Agaricus bisporus promoter of the glyceraldehyde-3-phosphate dehydrogenase $(g p d)$ gene to confer resistance to hygromycin B (HYG) (Figure 4B). Vector pBH-GFP-ActsPT further includes the eGFP gene from Aequorea victoria under the control of the ACT1 promoter and terminator of $A$. bisporus (Figure 4C). Another vector that encodes a fluorescent marker includes a Malassezia codon-optimized mCherry gene fused with the NAT marker, as in plasmid pAIM2, through a P2A sequence (Goh et al., 2020); P2A is a $2 \mathrm{~A}$ self-cleaving peptide derived from the porcine teschovirus-1 that was used to guarantee high expression of both the NAT and mCherry genes. These gene markers were also modified and reassembled to perform protein localization and chromatin immunoprecipitation (ChIP) through the generation of both N-terminal and C-terminal GFP fusion proteins, and 3xFLAG-tagged proteins (Ianiri et al., 2020; Sankaranarayanan et al., 2020).

One of the greatest advantages of AMT for Malassezia is its efficacy for approaches of both random insertional mutagenesis and targeted mutagenesis, which is not common for Basidiomycota fungi such as C. neoformans (McClelland et al., 2005). Insertional mutagenesis is carried out through AMT of a Malassezia species with one of the binary vectors 
A

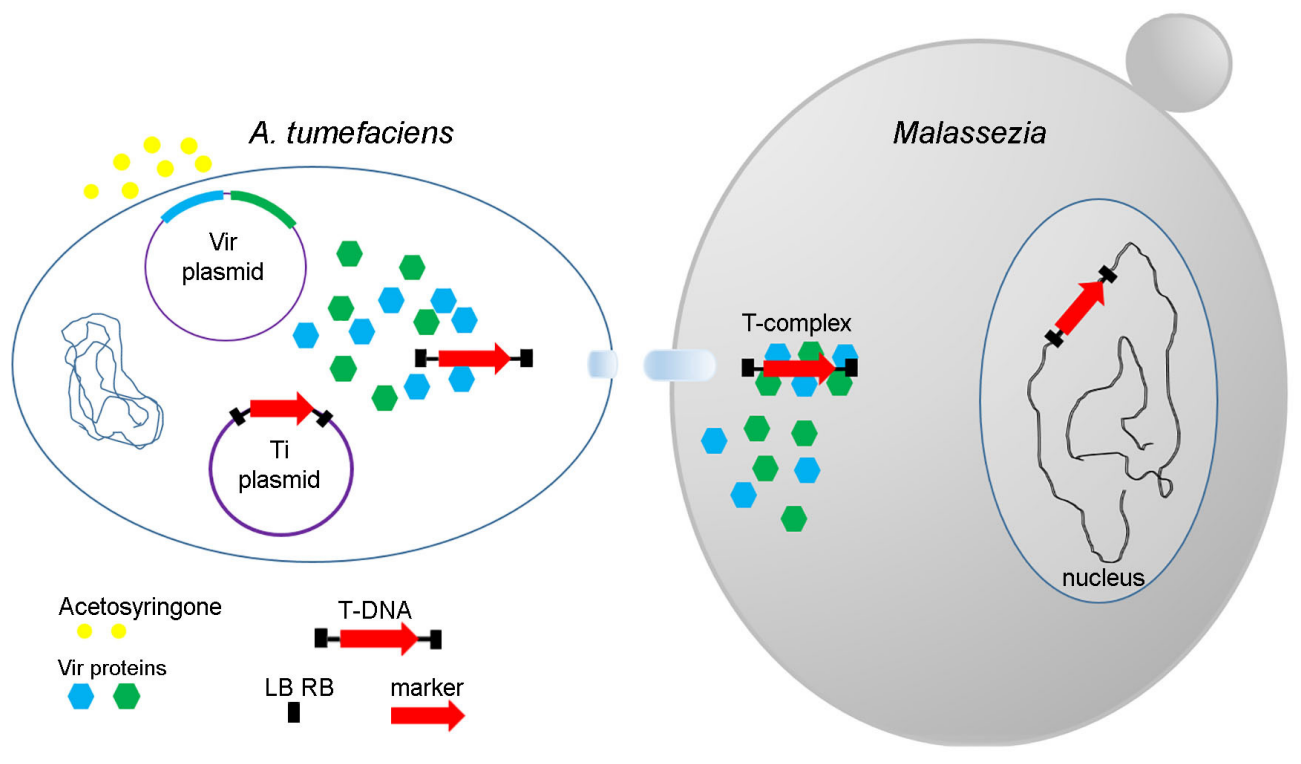

B

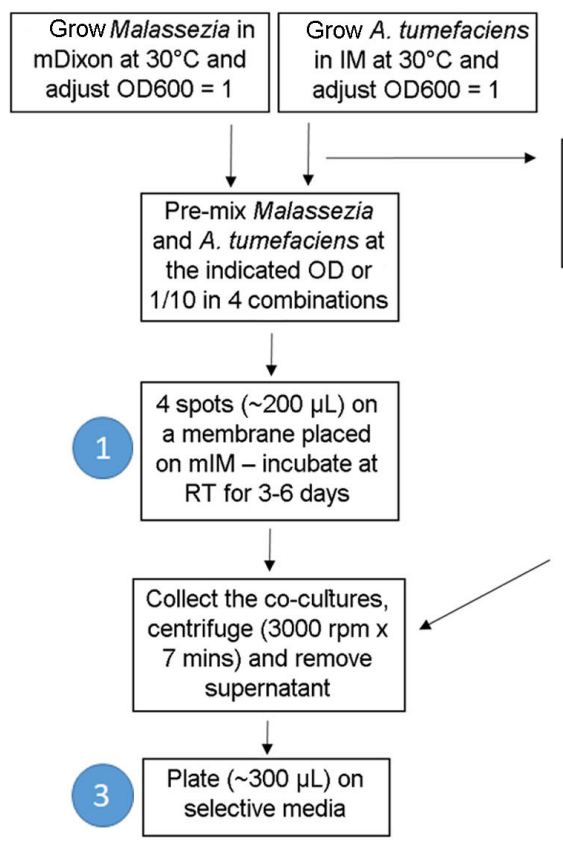

C

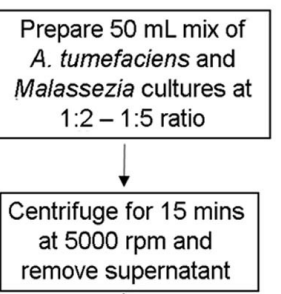

remove supernatant

spot $(500 \mu \mathrm{L}-1 \mathrm{~mL})$

on a membrane placed

on $\mathrm{mIM}$ - incubate at

RT for 3-6 days
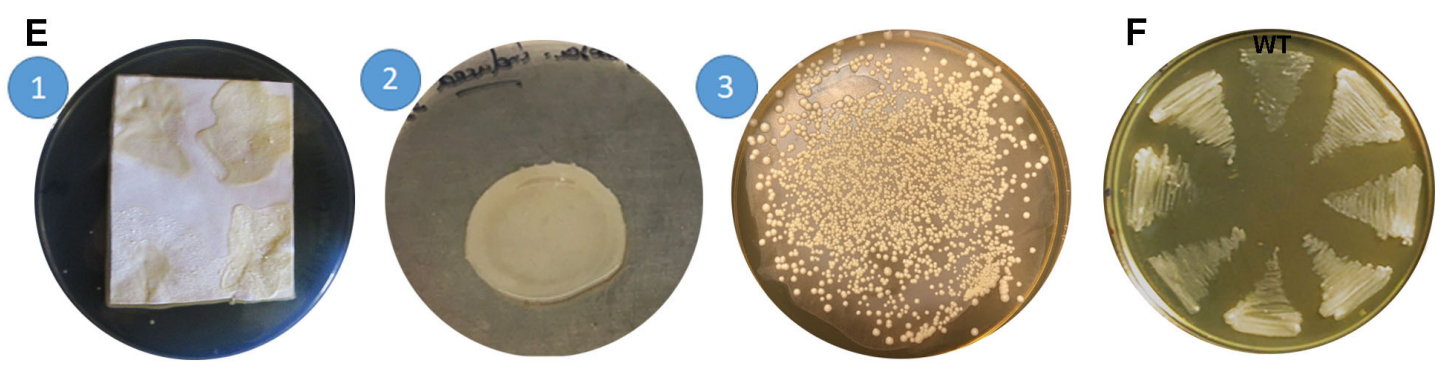

FIGURE $3 \mid$ A. tumefaciens-mediated transformation of Malassezia. (A) Schematic overview of the transformation process; see text for details. (B-D) Main steps of the protocols available for AMT of Malassezia according to laniri et al. (2016) (B), laniri et al. (2019) (C), and Celis et al. (2017) (D); the white numbers in the blue circle 
FIGURE 3 | reflect the corresponding step shown in images in (E). (E) Representative pictures of the Malassezia-A. tumefaciens co-incubation step (1) as described in laniri et al. (2016), (note the non-homogenous spots due to the presence of Tween that altered the physical proprieties of the IM agar) and (2) in Celis et al. (2017), and example of a highly efficient AMT of $M$. furfur with selection on NAT (3); the white numbers in the blue circles reflect the corresponding steps displayed in the charts shown in (B-D). (F) Growth of 7 representatives NAT-resistant M. furfur transformants on NAT selective media compared to the M. furfur WT strain.

\section{A}

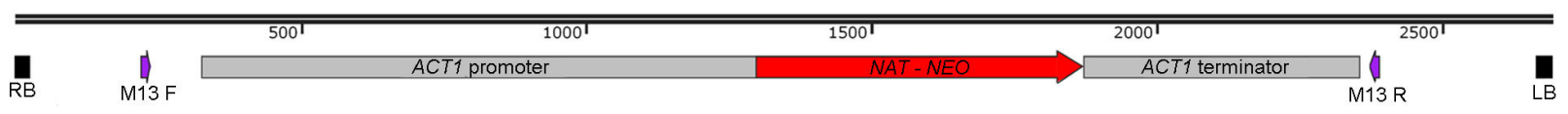

B

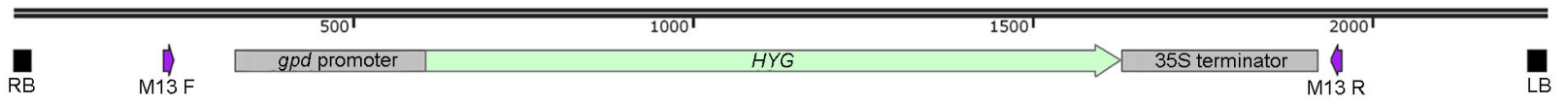

C

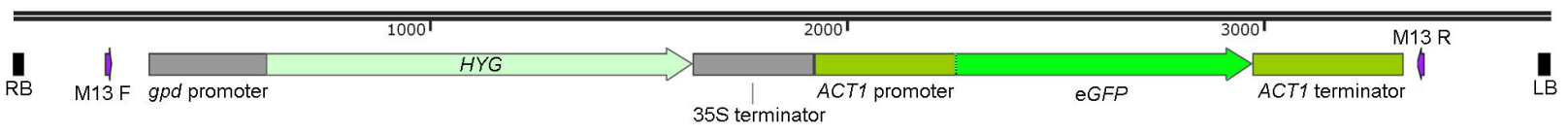

D

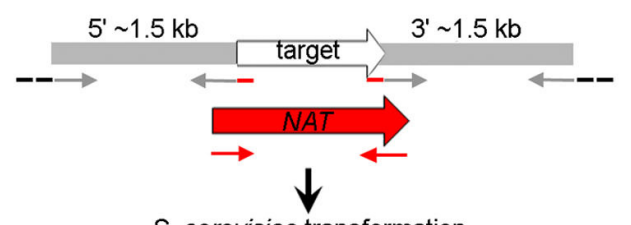

S. cerevisiae transformation

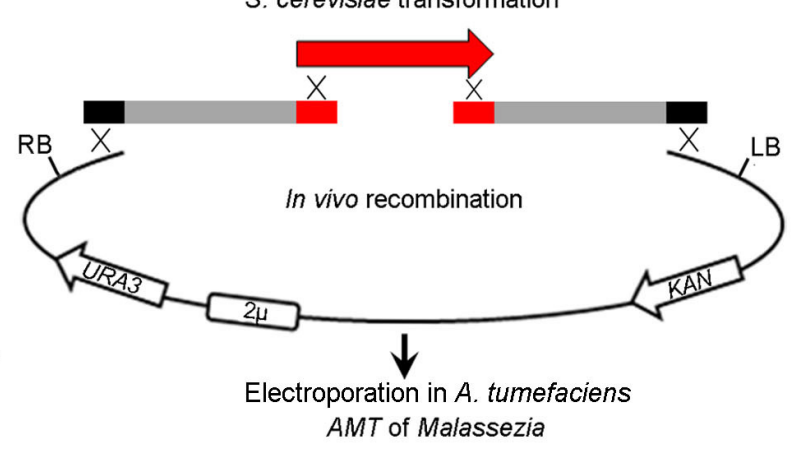

E

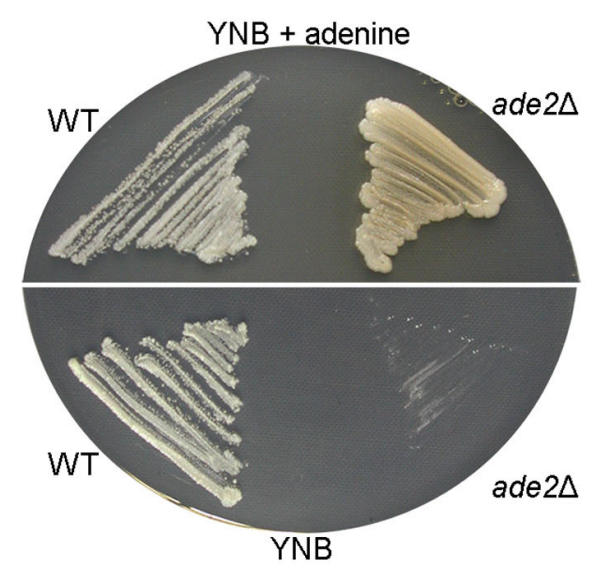

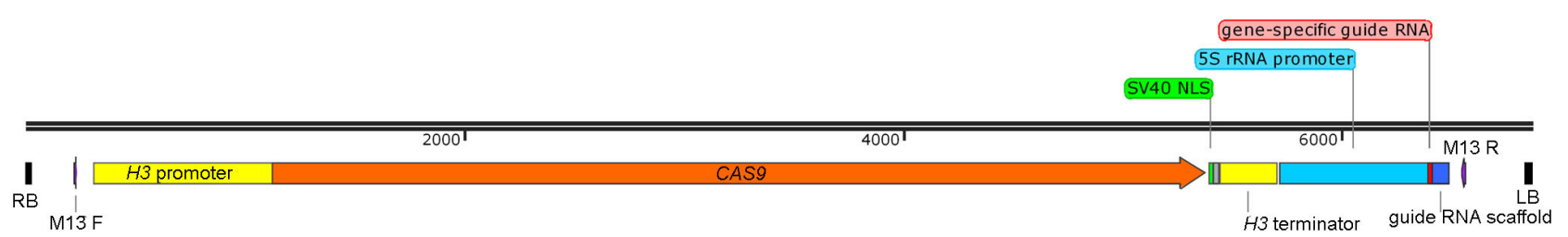

FIGURE 4 | Plasmids available for A. tumefaciens-mediated trasformation of Malassezia. (A) T-DNA of the plasmids pAIM2 and pAIM6 conferring resistance to NAT and NEO, respectively, as reported by laniri et al. (2016). (B) T-DNA of plasmid pBHg conferring resistance to HYG, and in (C) the same T-DNA including also a eGFP-expression cassette, as reported by Celis et al. (2017). (D) Strategy for in vivo recombination in S. cerevisiae developed to generate plasmids for targeted gene replacement in Malassezia; the schematic representation is adapted from laniri et al. (2016). (E) M. furfur ade2 $\Delta$ mutants generated through AMT in laniri et al. (2016); note the different growth pigmentation of the ade2 $\Delta$ mutant compared to the WT strain on mYNB supplemented or not with adenine ( $\mathrm{mYNB}$ stands for "modified YNB", which includes Tween 60, Tween 20, and ox-bile). (F) T-DNA of the plasmid pGI40 used by laniri et al. (2019) for transient CRISPR/Cas9-mediated targeted gene replacement in $M$. furfur.

described above, selection of stable drug-resistant transformants to be screened for a phenotype of interest, and identification of the genes that bear the random T-DNA insertion within the Malassezia genome; PCR-based techniques (inverse PCR and/or Splinkerette PCR) or whole-genome sequencing can be used to identify the site of insertion of the T-DNA (Idnurm 
et al., 2004; Ianiri et al., 2011; Ianiri and Idnurm, 2015). The random insertional mutagenesis approach was applied mainly in $M$. furfur and allowed the identification of (i) transformants unable to grow on minimal medium with T-DNA insertions in the TYR1 and ARG1 genes, (ii) a temperature-sensitive transformant with a T-DNA insertion in the promoter region of the JEN1 gene, (iii) a UV-sensitive transformant with a T-DNA insertion in the CDC55 gene, and (iv) several other transformants sensitive to the antifungal drug fluconazole, heavy metals, and cell wall stressing compounds (Ianiri et al., 2016, 2019). Insertional mutagenesis has the advantage that it can be used to discover novel genes and phenotypes; conversely, it has the disadvantage that transformants selected might have irregular and/or multiple T-DNA insertions and chromosomal rearrangements, factors that hinder the correct association between the mutated genes and the observed phenotypes. For such situations, in Malassezia the mutant phenotype can be confirmed through the de novo generation of a targeted deletion mutant for the identified gene, as we recently described (Ianiri et al., 2019).

Gene disruption mutagenesis involves the generation of a specific targeted mutant for a defined gene via homologous recombination. The first step is the generation of a gene deletion construct that includes $\sim 1-1.5 \mathrm{~kb}$ of sequence homologous to the regions flanking the gene of interest fused with a gene marker; when recipient organisms are transformed with this allele, homology with the flanking regions allows homologous recombination and the replacement of the target gene with the gene marker. For the use of AMT for targeted gene replacement, the gene deletion allele has to be assembled and cloned within the T-DNA of a binary vector. Although this can be achieved using several approaches, for gene deletion in Malassezia we developed a high-throughput strategy based on in vivo recombination in S. cerevisiae to simultaneously assemble and clone the gene replacement cassette within the T-DNA of a shuffle plasmid (Ianiri et al., 2017b). Briefly, three PCR fragments that include the gene marker gene and the $1.5 \mathrm{~kb}$ upstream $\left(5^{\prime}\right)$ and downstream $\left(3^{\prime}\right)$ regions flanking the target genes, and the KpnI-BamHI digested pGI3 plasmid, are transformed in S. cerevisiae wherein endogenous recombination is enabled by homologous regions between the PCR fragments and the digested plasmid (Ianiri et al., 2016), (Figure 4D).

In our first attempt, we tested the feasibility of AMT to generate $M$. furfur targeted mutants for the ADE2 gene, which was chosen because mutations in this gene result in a differential pigmentation compared to the WT hence allowing rapid evaluation of the results. We obtained several $M$. furfur ade2 $\Delta$ mutants that displayed adenine auxotrophy and a pigmentation that varied from light pink on rich media to yellow on minimal medium supplemented with adenine (Figure 4E), which is different from other yeasts such as $S$. cerevisiae (Zonneveld and van der Zanden, 1995). Subsequently, we applied this approach to study the function of the $M$. furfur laccase-encoding gene $L A C 1$ expected to play a role in pathogenesis (Ianiri et al., 2016), to elucidate the mechanisms of resistance of $M$. sympodialis to calcineurin inhibitors through mutations of the FKB1 and MSH2 genes (Ianiri et al., 2017a), and to demonstrate that the HGTmediated acquisition of the flavohemoglobin gene YHB1 in $M$. sympodialis resulted in a gain of function as described above
(Ianiri et al., 2020). Other mutated genes under investigation were the allergen-encoding gene MalaS8 in M. sympodialis, and the Rim101-alkaline pathway genes RIM101 and RRA1 in $M$. sympodialis and in M. furfur (unpublished data).

During the generation of these deletion mutants, we observed a lower rate of homologous recombination (HR) in $M$. furfur, about $\sim 50 \%$, compared to $M$. sympodialis, which had homologous recombination rates ranging between 90 and $100 \%$. While the mechanisms that control the rate of HR in these fungi are unknown and worthly of further investigation, in some cases we were unable to generate targeted mutants in $M$. furfur, especially for large genes. For these reasons, a novel CRISPR/Cas 9 system to increase the rate of HR and efficiently generate targeted mutants in $M$. furfur was developed. The system is based on co-transformation of $M$. furfur mediated by two A. tumefaciens strains to deliver both a CAS9-gRNA construct that induces double-strand DNA breaks, and a gene replacement allele that serves as a homology-directed repair template. The binary vector for Cas9 expression, pGI40, consists of the CAS9 gene fused with the histone $H 3$ promoter and terminator of $M$. sympodialis, followed by the $M$. sympodialis 5 S rRNA promoter fused with a gene-specific guide RNA, and a guide RNA scaffold (Ianiri et al., 2019), (Figure 4F). Using our AMT protocol, targeted deletion mutants for the M. furfur genes CDC55 and PDR10 were readily obtained with a HR rate of 100 and $83 \%$, respectively; note that PDR10 is large gene $(\sim 5 \mathrm{~kb})$ and such a high rate of HR was achieved using shorter flanking regions of $800 \mathrm{bp}$.

\section{CONCLUDING REMARKS}

Malassezia yeasts are attracting the interest of both basic and applied scientists because of their unique biological features, and importance in clinical and cosmetic settings. The availability of genome assemblies and robust tools for genetic manipulation allows both insertional mutagenesis and targeted gene replacement to be conducted. Results from these experiments can be combined with the increasing availability of transcriptomic data, with the possibility to focus further studies on novel key genes that characterize the Malassezia fungi. Moreover, from a more clinical perspective, tools for genetic manipulation can be combined with the use of host-pathogen interaction models, such as the easy-to-use wax moth larvae of Galleria mellonella (Torres et al., 2020), or a more complex murine skin model (Sparber and LeibundGut-Landmann, 2019; Sparber et al., 2019), enabling the characterization of both the fungal components that trigger skin damage and inflammation, and the inflammatory and antifungal response of the host to prevent fungal infection through immunological and molecular analyses of experimentally infected tissue.

\section{AUTHOR CONTRIBUTIONS}

GI and JH planned the review, read, and approved the final version. GI wrote the initial draft. All authors contributed to the article and approved the submitted version. 


\section{ACKNOWLEDGMENTS}

We thank our collaborators Dr. Kaustuv Syanal and Dr. Salomé LeibundGut-Landmann and people from their groups for invaluable discussions on Malassezia genomics and genetics that stimulated this review. We also thank Dr. Marco Coelho and Shelly Clancey from the Heitman lab for their contribution to several studies cited in this review. Last, we thank Alexander

\section{REFERENCES}

Aykut, B., Pushalkar, S., Chen, R., Li, Q., Abengozar, R., Kim, J. I., et al. (2019). The fungal mycobiome promotes pancreatic oncogenesis via activation of MBL. Nature 574, 264-267. doi: 10.1038/s41586-019-1608-2

Beceiro, A., Tomás, M., and Bou, G. (2013). Antimicrobial resistance and virulence: a successful or deleterious association in the bacterial world? Clin. Microbiol. Rev. 26, 185-230. doi: 10.1128/CMR.00059-12

Boekhout, T., and Bosboom, R. W. (1994). Karyotyping of Malassezia yeasts: taxonomic and epidemiological implications. Syst. Appl. Microbiol. 17, 146-153. doi: 10.1016/S0723-2020(11)80043-3

Boekhout, T., Kamp, M., and Gueho, E. (1998). Molecular typing of Malassezia species with PFGE and RAPD. Med. Mycol. 36, 365-372. doi: $10.1080 / 02681219880000581$

Bundock, P., den Dulk-Ras, A., Beijersbergen, A., and Hooykaas, P. J. (1995). Trans-kingdom T-DNA transfer from Agrobacterium tumefaciens to Saccharomyces cerevisiae. EMBO J. 14, 3206-3214. doi: 10.1002/j.1460-2075.1995.tb07323.x

Celis, A. M., Vos, A. M., Triana, S., Medina, C. A., Escobar, N., Restrepo, S., et al. (2017). Highly efficient transformation system for Malassezia furfur and Malassezia pachydermatis using Agrobacterium tumefaciens-mediated transformation. J. Microbiol. Methods 134, 1-6. doi: 10.1016/j.mimet.2017.01.001

Cho, Y.-J., Park, M., and Jung, W. H. (2019). Resequencing the genome of Malassezia restricta strain KCTC 27527. Microbiol. Resour. Announce. 8, e00213-00219. doi: 10.1128/MRA.00213-19

Clancey, S. A., Ruchti, F., LeibundGut-Landmann, S., Heitman, J., and Ianiri, G. (2019). A novel mycovirus evokes transcriptional rewiring in Malassezia and provokes host inflammation and an immunological response. bioRxiv [Preprint]. doi: 10.1101/2019.12.18.880518

de Hoog, S., Monod, M., Dawson, T., Boekhout, T., Mayser, P., and Gräser, Y. (2017). Skin fungi from colonization to infection. Microbiol. Spectrum 5, 855-871. doi: 10.1128/9781555819583.ch41

Drinnenberg, I. A., Fink, G. R., and Bartel, D. P. (2011). Compatibility with killer explains the rise of RNAi-deficient fungi. Science 333, 1592-1592. doi: 10.1126/science. 1209575

Findley, K., Oh, J., Yang, J., Conlan, S., Deming, C., Meyer, J. A., et al. (2013). Topographic diversity of fungal and bacterial communities in human skin. Nature 498, 367-370. doi: 10.1038/nature12171

Frandsen, R. J. (2011). A guide to binary vectors and strategies for targeted genome modification in fungi using Agrobacterium tumefaciens-mediated transformation. J. Microbiol. Methods 87, 247-262. doi: 10.1016/j.mimet.2011.09.004

Gaitanis, G., Magiatis, P., Hantschke, M., Bassukas, I. D., and Velegraki, A. (2012). The Malassezia genus in skin and systemic diseases. Clin. Microbiol. Rev. 25, 106-141. doi: 10.1128/CMR.00021-11

Gioti, A., Nystedt, B., Li, W., Xu, J., Andersson, A., Averette, A. F., et al. (2013). Genomic insights into the atopic eczema-associated skin commensal yeast Malassezia sympodialis. mBio 4, e00572-12. doi: 10.1128/mBio.00572-12

Glatz, M., Bosshard, P. P., Hoetzenecker, W., and Schmid-Grendelmeier, P. (2015). The role of Malassezia spp. in atopic dermatitis. J. Clin. Med. 4, 1217-1228. doi: $10.3390 / \mathrm{jcm} 4061217$

Goh, J. P., Ianiri, G., Heitman, J., and Dawson, T. L. (2020). Expression of a Malassezia codon optimized mCherry fluorescent protein in a bicistronic vector. Front. Cell. Infect. Microbiol. 10:367. doi: 10.3389/fcimb.2020. 00367
Idnurm and Thomas Dawson for their encouragement to perform research on Malassezia. Research on Malassezia in the Heitman's lab has been supported by NIH/NIAID R37 award AI39115-23, R01 award AI50113-16 awarded to JH, and by the Duke Microbiome Center to GI. JH is a CoDirector and Fellow of the CIFAR Program Fungal Kingdom: Threats \& Opportunities.

Guillot, J., and Bond, R. (2020). Malassezia yeasts in veterinary dermatology: an updated overview. Front. Cell. Infect. Microbiol. 10:79. doi: $10.3389 /$ fcimb. 2020.00079

Ianiri, G., Applen Clancey, S., Lee, S. C., and Heitman, J. (2017a). FKBP12dependent inhibition of calcineurin mediates immunosuppressive antifungal drug action in Malassezia. mBio 8, e01752-17. doi: 10.1128/mBio.01752-17

Ianiri, G., Averette, A. F., Kingsbury, J. M., Heitman, J., and Idnurm, A. (2016). Gene function analysis in the ubiquitous human commensal and pathogen Malassezia genus. mBio 7, e01853-16. doi: 10.1128/mBio.01853-16

Ianiri, G., Boyce, K. J., and Idnurm, A. (2017b). Isolation of conditional mutations in genes essential for viability of Cryptococcus neoformans. Curr. Genet. 63, 519-530. doi: 10.1007/s00294-016-0659-2

Ianiri, G., Coelho, M. A., Ruchti, F., Sparber, F., McMahon, T. J., Fu, C., et al. (2020). HGT in the human and skin commensal Malassezia: A bacterially derived flavohemoglobin is required for NO resistance and host interaction. Proc. Natl. Acad. Sci. U. S. A. 117:15884-15894. doi: 10.1073/pnas.2003473117.

Ianiri, G., Dagotto, G., Sun, S., and Heitman, J. (2019). Advancing functional genetics through Agrobacterium-mediated insertional mutagenesis and CRISPR/Cas9 in the commensal and pathogenic yeast Malassezia. Genetics 212, 1163-1179. doi: 10.1534/genetics.119.302329

Ianiri, G., and Idnurm, A. (2015). Essential gene discovery in the basidiomycete Cryptococcus neoformans for antifungal drug target prioritization. mBio 6, e02334-14. doi: 10.1128/mBio.02334-14

Ianiri, G., Wright, S. A., Castoria, R., and Idnurm, A. (2011). Development of resources for the analysis of gene function in Pucciniomycotina red yeasts. Fungal Genet. Biol. 48, 685-695. doi: 10.1016/j.fgb.2011.03.003

Idnurm, A., Bailey, A. M., Cairns, T. C., Elliott, C. E., Foster, G. D., Ianiri, G., et al. (2017). A silver bullet in a golden age of functional genomics: the impact of Agrobacterium-mediated transformation of fungi. Fungal Biol. Biotechnol. 4:6. doi: 10.1186/s40694-017-0035-0

Idnurm, A., Reedy, J. L., Nussbaum, J. C., and Heitman, J. (2004). Cryptococcus neoformans virulence gene discovery through insertional mutagenesis. Eukaryotic Cell 3, 420-429. doi: 10.1128/EC.3.2.420-429.2004

Limon, J. J., Tang, J., Li, D., Wolf, A. J., Michelsen, K. S., Funari, V., et al. (2019). Malassezia is associated with Crohn's disease and exacerbates colitis in mouse models. Cell Host Microbe 25, 377-388. doi: 10.1016/j.chom.2019.01.007

Lorch, J. M., Palmer, J. M., Vanderwolf, K. J., Schmidt, K. Z., Verant, M. L., Weller, T. J., et al. (2018). Malassezia vespertilionis sp. nov.: a new cold-tolerant species of yeast isolated from bats. Persoonia Mol. Phylog. Evol. Fungi 41, 56-70. doi: $10.3767 /$ persoonia.2018.41.04

McClelland, C. M., Chang, Y. C., and Kwon-Chung, K. J. (2005). High frequency transformation of Cryptococcus neoformans and Cryptococcus gattii by Agrobacterium tumefaciens. Fungal Genet. Biol. 42, 904-913. doi: 10.1016/j.fgb.2005.07.003

Michielse, C. B., Hooykaas, P. J., van den Hondel, C. A. M. J. J., and Ram, A. F. (2005). Agrobacterium-mediated transformation as a tool for functional genomics in fungi. Curr. Genet. 48, 1-17. doi: 10.1007/s00294-005-0578-0

Morand, S. C., Bertignac, M., Iltis, A., Kolder, I. C. R. M., Pirovano, W., Jourdain, R., et al. (2019). Complete genome sequence of Malassezia restricta CBS 7877, an opportunist pathogen involved in dandruff and seborrheic dermatitis. Microbiol. Resour. Announce. 8, e01543-18. doi: 10.1128/MRA.01543-18

Mosqueda, N., Gato, E., Roca, I., Lopez, M., de Alegria, C. R., Fernandez Cuenca, F., et al. (2014). Characterization of plasmids carrying the blaOXA24/40 carbapenemase gene and the genes encoding the AbkA/AbkB proteins of a toxin/antitoxin system. J. Antimicrob. Chemother. 69, 2629-2633. doi: $10.1093 / \mathrm{jac} / \mathrm{dku} 179$ 
Park, M., Cho, Y.-J., Kim, D., Yang, C.-S., Lee, S. M., Dawson, T. L., et al. (2019). A novel totivirus alters gene expression and vacuolar morphology in Malassezia cells and induces a TLR3-mediated inflammatory immune response. bioRxiv [Preprint]. doi: 10.1101/2019.12.17.880526

Park, M., Cho, Y. J., Lee, Y. W., and Jung, W. H. (2017). Whole genome sequencing analysis of the cutaneous pathogenic yeast Malassezia restricta and identification of the major lipase expressed on the scalp of patients with dandruff. Mycoses 60, 188-197. doi: 10.1111/myc.12586

Puig, L., Bragulat, M. R., Castellá, G., and Cabañes, F. J. (2017). Characterization of the species Malassezia pachydermatis and re-evaluation of its lipid dependence using a synthetic agar medium. PLOS ONE 12:e179148. doi: 10.1371 /journal.pone.0179148

Sankaranarayanan, S. R., Ianiri, G., Coelho, M. A., Reza, M. H., Thimmappa, B. C., Ganguly, P., et al. (2020). Loss of centromere function drives karyotype evolution in closely related Malassezia species. elife 9:e53944. doi: 10.7554/eLife.53944.sa2

Saunders, C. W., Scheynius, A., and Heitman, J. (2012). Malassezia fungi are specialized to live on skin and associated with dandruff, eczema, and other skin diseases. PLoS Pathog. 8:e1002701. doi: 10.1371/journal.ppat.1002701

Schuster, M., Schweizer, G., and Kahmann, R. (2018). Comparative analyses of secreted proteins in plant pathogenic smut fungi and related basidiomycetes. Fungal Genet. Biol. 112, 21-30. doi: 10.1016/j.fgb.2016.12.003

Soret, P., Vandenborght, L.-E., Francis, F., Coron, N., Enaud, R., Avalos, M., et al. (2020). Respiratory mycobiome and suggestion of inter-kingdom network during acute pulmonary exacerbation in cystic fibrosis. Sci. Rep. 10:3589. doi: 10.1038/s41598-020-60015-4

Sparber, F., De Gregorio, C., Steckholzer, S., Ferreira, F. M., Dolowschiak, T., Ruchti, F., et al. (2019). The skin commensal yeast Malassezia triggers a type 17 response that coordinates anti-fungal immunity and exacerbates skin inflammation. Cell Host Microbe 25, 389-403. doi: 10.1016/j.chom.2019.02.002

Sparber, F., and LeibundGut-Landmann, S. (2019). Infecting mice with Malassezia spp. to study the fungus-host interaction. J. Visualized Exp. doi: 10.3791/60175

Tannous, J., Snini, S. P., El Khoury, R., Canlet, C., Pinton, P., Lippi, Y., et al. (2017). Patulin transformation products and last intermediates in its biosynthetic pathway, E- and Z-ascladiol, are not toxic to human cells. Arch. Toxicol. 91, 2455-2467. doi: 10.1007/s00204-016-1900-y

Theelen, B., Cafarchia, C., Gaitanis, G., Bassukas, I. D., Boekhout, T., and Dawson, T. L., Jr. (2018). Malassezia ecology, pathophysiology, and treatment. Med. Mycol. 56(Suppl. 1), S10-S25. doi: 10.1093/mmy/myx134

Torres, M., Pinzón, E. N., Rey, F. M., Martinez, H., Parra Giraldo, C. M., and Celis Ramírez, A. M. (2020). Galleria mellonella as a novelty in vivo model of host-pathogen interaction for Malassezia furfur CBS 1878 and Malassezia pachydermatis CBS 1879. Front. Cell. Infect. Microbiol. 10:199. doi: 10.3389/fcimb.2020.00199

Triana, S., González, A., Ohm, R. A., Wösten, H. A. B., de Cock, H., Restrepo, S., et al. (2015). Draft genome sequence of the animal and human pathogen
Malassezia pachydermatis strain CBS 1879. Genome Announce. 3, e01197-15. doi: 10.1128/genomeA.01197-15

Velegraki, A., Cafarchia, C., Gaitanis, G., Iatta, R., and Boekhout, T. (2015). Malassezia infections in humans and animals: pathophysiology, detection, and treatment. PLoS Pathog. 11:e1004523. doi: 10.1371/journal.ppat. 1004523

Vilhelmsson, M., Zargari, A., Crameri, R., Rasool, O., Achour, A., Scheynius, A., et al. (2007). Crystal structure of the major Malassezia sympodialis allergen Mala S 1 reveals a $\beta$-propeller fold: a novel fold among allergens. J. Mol. Biol. 369, 1079-1086. doi: 10.1016/j.jmb.2007.04.009

Wang, Q. M., Theelen, B., Groenewald, M., Bai, F. Y., and Boekhout, T. (2014). Moniliellomycetes and Malasseziomycetes, two new classes in Ustilaginomycotina. Persoonia 33, 41-47. doi: 10.3767/003158514X6 82313

Wu, G., Zhao, H., Li, C., Rajapakse, M. P., Wong, W. C., Xu, J., et al. (2015). Genus-wide comparative genomics of Malassezia delineates its phylogeny, physiology, and niche adaptation on human skin. Front. Genet. 11:e1005614. doi: 10.1371/journal.pgen.1005614

Xu, J., Saunders, C. W., Hu, P., Grant, R. A., Boekhout, T., Kuramae, E. E., et al. (2007). Dandruff-associated Malassezia genomes reveal convergent and divergent virulence traits shared with plant and human fungal pathogens. Proc. Natl. Acad. Sci. U.S.A. 104, 18730-18735. doi: 10.1073/pnas.07067 56104

Zargari, A., Selander, C., Rasool, O., Ghanem, M., Gadda, G., Crameri, R., et al. (2007). Mala S 12 is a major allergen in patients with atopic eczema and has sequence similarities to the GMC oxidoreductase family. Allergy 62, 695-703. doi: 10.1111/j.1398-9995.2006.01291.x

Zhu, Y., Engstrom, P. G., Tellgren-Roth, C., Baudo, C. D., Kennell, J. C., Sun, S., et al. (2017). Proteogenomics produces comprehensive and highly accurate protein-coding gene annotation in a complete genome assembly of Malassezia sympodialis. Nucleic Acids Res. 45, 2629-2643. doi: 10.1093/nar/gkx006

Zonneveld, B. J., and van der Zanden, A. L. (1995). The red ade mutants of Kluyveromyces lactis and their classification by complementation with cloned ADE1 or ADE2 genes from Saccharomyces cerevisiae. Yeast 11, 823-827. doi: 10.1002/yea.320110904

Conflict of Interest: The authors declare that the research was conducted in the absence of any commercial or financial relationships that could be construed as a potential conflict of interest.

Copyright (c) 2020 Ianiri and Heitman. This is an open-access article distributed under the terms of the Creative Commons Attribution License (CC BY). The use, distribution or reproduction in other forums is permitted, provided the original author(s) and the copyright owner(s) are credited and that the original publication in this journal is cited, in accordance with accepted academic practice. No use, distribution or reproduction is permitted which does not comply with these terms. 